\title{
The minimal length uncertainty and the nonextensive thermodynamics
}

\author{
Homa Shababi and Pouria Pedram* \\ Department of Physics, Science and Research Branch, Islamic Azad University, Tehran, Iran
}

(Dated: October 15, 2018)

\begin{abstract}
In this paper, we study the thermodynamics of quantum harmonic oscillator in the Tsallis framework and in the presence of a minimal length uncertainty. The existence of the minimal length is motivated by various theories such as string theory, loop quantum gravity, and black-hole physics. We analytically obtain the partition function, probability function, internal energy, and the specific heat capacity of the vibrational quantum system for $1<q<\frac{3}{2}$ and compare the results with those of Tsallis and Boltzmann-Gibbs statistics without the minimal length scale.
\end{abstract}

PACS numbers: $04.60 . \mathrm{Bc}$

Keywords: Minimal length, Generalized uncertainty principle, Nonextensive thermodynamics

\section{INTRODUCTION}

Various approaches to quantum gravity such as string theory [1-7], noncommutative geometry [8], and loop quantum gravity [9] motivate the existence of a minimal measurable length. These theories argue that near the Planck scale, the Heisenberg Uncertainty Principle (HUP) should be replaced by the so-called Generalized Uncertainty Principle (GUP) (see Refs. [1 7] and references therein). The effects of gravity play an important role in the limit of small distances around the Planck length, $\ell_{P l}=\sqrt{G \hbar / c^{3}} \approx 10^{-35} \mathrm{~m}$, where $G$ is Newton's constant, $\hbar$ is the Planck constant, and $c$ is the speed of light. Equivalently, these effects are dominant in the limit of high energies near the Planck energy $E_{P l}=\sqrt{\hbar c^{5} / G} \approx 1.956 \times 10^{9} \mathrm{~J}$.

In nonrelativistic quantum mechanics, a particle with an arbitrary energy can be accurately localized and its exact location can be obtained. According to the quantum filed theory and based on Heisenberg Uncertainty Principle, only particles with infinite energy can be localized. However, in quantum gravity theories that try to combine the effects of gravity with quantum mechanics, the localization of particles completely disappear. In general relativity, it is possible to access the path of particles that is impossible to obtain in quantum mechanics. In fact, in quantum mechanics determination of exact position of particles requires infinite momentum uncertainty. Now, in order to achieve the ultimate theory of quantum gravity, one of the results of combination of gravity and quantum mechanics is introducing the minimal measurable length of the order of the Planck length. In this case, particles lose their point-like description. Thus, to find the position of particles, a minimal length uncertainty proportional to the Planck length should be taken into account and the sharp localization condition for particles is relaxed [10].

Moreover, some Gedanken experiments in black-hole physics and noncommutativity of the spacetime manifold predict the existence of a minimal observable distance of the order of the Planck length [11 15]. The generalization of ordinary uncertainty relation by incorporating quantum gravity effects requires a modification of the Hamiltonian of various physical systems which has been studied in the literature [16-23].

According to recent statistical approaches, standard thermodynamics seems to be not suitable for studying all systems including the behavior of complex systems which are governed by the nonextensive statistics [24 28]. A nonextensive statistical mechanics which is proposed by Tsallis [29] and inspired by the probabilistic description of multifractal geometries, generalizes all thermodynamical quantities such as the partition function and the internal energy with the entropic index $q$ that plays an important role in this framework. Moreover, in the limit $q \rightarrow 1$ it gives the well-known Boltzmann-Gibbs statistics.

Notice that the nonextensive statistics could be more useful than the traditional Boltzmann-Gibbs statistics in many situations. The theory behind the Boltzmann-Gibbs entropy implies a strong dependence on initial conditions. However, most materials behave quite independently of the initial conditions. In other words, the usage of the wellknown BG statistical mechanics occasionally fails and leads to serious difficulties. In this regards, we can mention long-range interactions, e.g., $d=3$ gravitation [30], long-range microscopic memory, e.g., nonmarkovian stochastic processes [31, 32], and conservative or dissipative systems multifractal-like structures [33]. More examples include granular systems [34], Levy anomalous diffusion [35], pure-electron plasma two-dimensional turbulence [36], phonon-

*Electronic address: p.pedram@srbiau.ac.ir 
electron anomalous thermalization in ion-bombarded solids [37], solar neutrinos [38], peculiar velocities of galaxies [39], and inverse bremsstrahlung in plasma [40] and black-holes [41].

During the last decade, Tsallis statistics has attracted much attention in the literature [42 51]. Among these investigations, we can mention the harmonic oscillator in both classical and quantum domains [49], the phase space of Tsallis entropy for the harmonic oscillator [52], the quantum entanglement of maximum Tsallis entropy state [53], path integral approach to the nonextensive canonical density matrix [54], the hydrogen atom in three dimensions [55], and the sensitivity of the population state to the value of $q$ for a two-state system [56]. Moreover, Tsallis entropy is compared to two different entropies, namely, Renyi entropy $S_{q}^{R}=\frac{1}{1-q} \ln \sum_{n}\left(p_{n}\right)^{q}$, and the normalized Tsallis entropy, $S_{q}^{N T}=\frac{1}{1-q}\left(1-\frac{1}{\sum_{n} p_{n}^{q}}\right)$, which is shown that the Tsallis entropy is more stable [57]. Recently, some authors have investigated the exact vibrational partition function for quantum nonextensive harmonic oscillator [58]. They showed that for all $T$ and $1<q<2$ the inequality $Z>Z^{*}$ holds and $Z-Z^{*}$ increases as $q$ and $T$ grow up where $Z$ and $T$ represent the partition function and temperature, respectively. Here and throughout the paper the symbol $*$ denotes the quantities obtained in the extensive statistics, i.e., Boltzmann-Gibbs thermodynamics.

Nowadays, it is believed that the minimal length scale naturally appears in various areas of high energy physics $[9]$. In particular, the effects of minimal length scale on thermodynamics of various physical systems have been studied in the literature (see, e.g., Refs. [16 23]). At this point, it is worth explaining why the idea of minimal length scale can be incorporated in thermodynamics. Consider a system which cannot be probed to distances smaller than some length scale. This phenomenon can be an intrinsic property of the system such as the minimal length scale in high energy physics which is of the order of the Planck length [2, 6, 9, 10, 59 63. On the other hand, it can be due to the size effect of the measurement apparatus. For instance, in finance as a complex system, the existence of a minimal length scale is necessary due to the fact that the stock price is indeed a discrete variable [64]. In thermodynamics, the gas particles have a nonzero size of the order of the atom's radius. So, these particles cannot probe distances smaller than their finite size which is indeed the minimal length scale of the system. Here, we use a framework that is compatible with this minimal length scale.

In this paper, we study the thermodynamics of the quantum harmonic oscillator in the presence of a minimal length and in the nonextensive Tsallis statistics. Although, other forms of GUP which imply both minimal measurable length and maximal momentum could be considered [65 69], to obtain exact solutions we only consider the existence of a minimal length. Notice that at ordinary distances the effects of gravity are not dominant and the ordinary physical laws can be used without difficulty. However, at small distances of the order of Planck length, the effects of gravity are considerable and the modification of physical laws would be required. The usage of the Tsallis statistics is based on the fact that physical systems with long-range interactions, long-time memories, and multifractal structures can hardly be treated within the traditional BG statistical framework. Therefore, the application of nonextensive statistical models is unavoidable for these systems.

The aim of our investigation is to analyze the behavior of a nonextensive thermodynamical system in the framework of the quantum gravity. Also, the modification of the thermodynamical variables in the presence of the minimal length uncertainty is addressed. The nonextensive statistical model contains the effect of multifractal geometry and therefore the concept of discrete geometric constructions can be essentially derived from multifractal geometry. However, the probabilities in nonextensive models depend on the energy spectrum of the system. In fact, the statistical model does not obtain the energy spectrum, it only use them to construct the partition function. So, when an statistical model agrees with the concept of discrete geometric constructions, the energy spectrum should be also obtained from a framework which agrees with this concept. In other words, a consistent way to find the nonextensive partition function is to use the GUP-corrected energy spectrum as the input of the nonextensive model which both imply the discrete geometry.

Here, the vibrational partition function, probability function, internal energy, and the specific heat capacity are obtained for $1<q<\frac{3}{2}$. We also compare our results with the corresponding quantities in the absence of minimal length [58] and with the Boltzmann-Gibbs thermodynamics. Note that the black-body radiation in Tsallis statistics and in the presence of the minimal length can also be treated using this model.

\section{QUANTUM OSCILlatoR IN THE PRESENCE OF A MINIMAL LENGTH SCALE}

In ordinary quantum mechanics, Heisenberg uncertainty principle allows us to measure the position and momentum of a particle separately with an arbitrary precision. But in the presence of a minimal value for the position measurement, the Heisenberg uncertainty relation should be modified. One way to incorporate the minimal length is to generalize the Heisenberg uncertainty principle as follows [10]

$$
\Delta X \Delta P \geq \frac{\hbar}{2}\left(1+\beta\left[(\Delta P)^{2}+\langle P\rangle^{2}\right]\right)
$$


where $\beta$ is the deformation parameter and defined as $\beta=\beta_{0} /\left(M_{P l} c\right)^{2}$ which $M_{P l}$ is the Planck mass and $\beta_{0}$ is of the order of unity. To show this, note that the absolute minimal measurable length is obtained by saturating the above inequality. So, we find $(\Delta X)_{\min }=\hbar \sqrt{\beta} \sqrt{1+\beta\langle P\rangle^{2}}$ and the absolute minimal measurable length is given by $(\Delta X)_{\min }=\hbar \sqrt{\beta}$ for $\langle P\rangle=0$. In the context of string theory this length can be interpreted as the length of the string and therefore the string's length is proportional to the square root of the GUP parameter. In thermodynamics, this minimal length is given by the size of the atoms where distances smaller than $(\Delta X)_{\min }$ cannot be probed by the particles. Notice that, since we have $(\Delta X)_{\min }=\sqrt{\beta_{0}} \ell_{P l}$, the minimal length scale is not essentially equal to the Planck length, but it is of the order of the Planck length. So, $(\Delta X)_{\min }$ could be smaller or bigger than the Planck length upon choosing $\beta_{0}<1$ or $\beta_{0}>1$. The exact value for $\beta_{0}$ is hoped to be obtained in the future experiments. In one dimension, the generalized uncertainty relation leads to the following deformed commutation relation

$$
[X, P]=i \hbar\left(1+\beta P^{2}\right) \text {. }
$$

According to Refs. [19, 70], $X$ and $P$ can be written in terms of ordinary position and momentum operators as

$$
X=x, \quad P=\frac{\tan (\sqrt{\beta} p)}{\sqrt{\beta}},
$$

where $[x, p]=i \hbar$. Moreover, all Hamiltonians will be modified due to the deformed commutation relation. In this formally self-adjoint representation, the Hamiltonian $H=\frac{P^{2}}{2 m}+V(X)$ can be expressed as

$$
H=\frac{\tan ^{2}(\sqrt{\beta} p)}{2 \beta m}+V(x) .
$$

Here, we study the thermodynamics of the quantum harmonic oscillator which its Hamiltonian is given by $H=$ $\frac{P^{2}}{2 m}+\frac{1}{2} m \omega^{2} X^{2}$. So, using the representation (3), the generalized Schrödinger equation in momentum space reads

$$
-\frac{1}{2} m \hbar^{2} \omega^{2} \frac{d^{2} \phi(p)}{d p^{2}}+\frac{\tan ^{2}(\sqrt{\beta} p)}{2 m \beta} \phi(p)=E \phi(p) .
$$

Using the new variable $\xi=\sqrt{\beta} p$, the above equation can be written as

$$
\frac{d^{2} \phi(\xi)}{d \xi^{2}}+\left(\epsilon-\frac{V}{\cos ^{2} \xi}\right) \phi(\xi)=0
$$

where $V=(m \beta \hbar \omega)^{-2}$ and $\epsilon=V(1+2 m \beta E)$. Now, taking

$$
\phi_{n}(\xi)=P_{n}(s) \cos ^{\lambda} \xi
$$

leads to

$$
\left(1-s^{2}\right) \frac{d^{2} P_{n}(s)}{d s^{2}}-s(1+2 \lambda) \frac{d P_{n}(s)}{d s}+\left(\epsilon-\lambda^{2}\right) P_{n}(s)=0,
$$

where $s=\sin \xi$ and

$$
V=\lambda(\lambda-1), \quad \lambda=\frac{1}{2}\left[1+\sqrt{1+\frac{4}{m^{2} \beta^{2} \hbar^{2} \omega^{2}}}\right] .
$$

The solutions of the above equation for $\epsilon=(n+\lambda)^{2}$ are given by the Gegenbauer polynomials $C_{n}^{\lambda}(s)$. Therefore, the exact solutions read

$$
\begin{aligned}
\phi_{n}(p) & =N_{n} C_{n}^{\lambda}(\sin (\sqrt{\beta} p)) \cos ^{\lambda}(\sqrt{\beta} p), \\
E_{n} & =\hbar \omega\left(n+\frac{1}{2}\right)\left(\sqrt{1+\gamma^{2} / 4}+\gamma / 2\right)+\frac{1}{2} \hbar \omega \gamma n^{2},
\end{aligned}
$$

for $n=0,1,2, \ldots$. Here $\gamma=m \beta \hbar \omega, N_{n}$ is the normalization coefficient, and the Gegenbauer polynomials are defined as 71 .

$$
C_{n}^{\lambda}(s)=\sum_{k=0}^{[n / 2]}(-1)^{k} \frac{\Gamma(n-k+\lambda)}{\Gamma(\lambda) k !(n-2 k) !}(2 s)^{n-2 k} .
$$

Note that in the absence of minimal length scale $(\beta \sim \gamma \rightarrow 0)$, the ordinary energy spectrum of the quantum harmonic oscillator is obtained, i.e., $E_{n}=\hbar \omega\left(n+\frac{1}{2}\right)$. 


\section{THERMODYNAMICS IN THE TSALLIS FRAMEWORK}

The entropy of Tsallis statistics is given by [29]

$$
S_{q}=k \frac{1-\sum_{n} p_{n}^{q}}{q-1}
$$

where $p_{n}$ is the probability of the $n$th microstate, $k$ is a positive constant, and $q$ is the entropic index. Using two constrains $\sum_{n=1}^{w} p_{n}=1$ and $\sum_{n=1}^{w} E_{n} p_{n}^{q}=U_{q}$ that maximize Eq. (13) where $w$ is the total number of microstates of the system [29, 72], the generalized statistical probability $p_{n}$ and the partition function $Z_{q}$ are given by

$$
\begin{aligned}
& p_{n}=\frac{\left[1-b(1-q) E_{n}\right]^{\frac{1}{1-q}}}{Z_{q}}, \\
& Z_{q}=\sum_{n=0}^{\infty}\left[1-b(1-q) E_{n}\right]^{\frac{1}{1-q}},
\end{aligned}
$$

where $b^{-1}=k T$ is the Lagrange parameter related to the internal energy $U_{q}$, and $E_{n}$ is the energy of the $n$th quantum state. Now, using Eq. (11) up to the first order of $\gamma$, the vibrational partition function reads

$$
Z_{v i b, q}=\sum_{n=0}^{\infty} \frac{1}{\left[1+b(q-1)\left(n+\frac{1}{2}\right) \hbar \omega\left(1+\frac{\gamma}{2}\right)+\frac{b \hbar \omega \gamma(q-1)}{2} n^{2}\right]^{\frac{1}{q-1}}}
$$

where $n$ is the quantum number and $\omega$ is the frequency of the harmonic oscillator.

Notice that, the convergence of the above equation implies $q>1$. Now, since $\gamma$ is a small parameter, up to the first order we have

$$
Z_{v i b, q}=[b(q-1) \hbar \omega]^{\frac{1}{1-q}} \sum_{n=0}^{\infty} \frac{1}{(n+a)^{s}}\left[1-\frac{\gamma s}{2}\left(\frac{n^{2}+n+\frac{1}{2}}{n+a}\right)\right]
$$

where $s=\frac{1}{q-1}$ and $a=\frac{1}{b(q-1) \hbar \omega}+\frac{1}{2}$. Consequently, using the Hurwits zeta function $\zeta(s, a)=\sum_{n=0}^{\infty} \frac{1}{(n+a)^{s}}$ and after some algebra, the analytic relation for the partition function is given by

$$
Z_{v i b, q}=Z_{0}+\gamma s[b(q-1) \hbar \omega]^{\frac{1}{1-q}}\left\{\left(a-\frac{1}{2}\right) \zeta(s, a)-\frac{1}{2} \zeta(s-1, a)-\left(\frac{a^{2}}{2}-\frac{a}{2}+\frac{1}{4}\right) \zeta(s+1, a)\right\}
$$

which $Z_{0} \equiv Z_{v i b, q}(\gamma=0=\beta)$ is the vibrational partition function of quantum harmonic oscillator in the absence of minimal length scale [58], namely $Z_{0}=[b(q-1) \hbar \omega]^{\frac{1}{1-q}} \zeta(s, a)$. Taking into account two conditions imposed by the definition of Hurwits zeta function, i.e., $s>1$ and $a>0$, the validity of Eq. (18) implies the inequality $1<q<\frac{3}{2}$. Also, in the limit of $q \rightarrow 1$, Eq. (18) gives the corresponding Boltzmann-Gibbs partition function in the presence of the minimal length scale [19]. For our case, the nonextensive probability function can be expressed as

$$
p_{n}=\frac{\left[1+b(q-1)\left(n+\frac{1}{2}\right) \hbar \omega\left(1+\frac{\gamma}{2}\right)+\frac{b \hbar \omega \gamma(q-1)}{2} n^{2}\right]^{\frac{1}{1-q}}}{Z_{v i b, q}} .
$$

To investigate the results, we plotted the probability functions versus the quantum number for two temperatures in Figs. 1 and 2 For $T=2$, as it is shown in Fig. 11, we have $p_{n}^{*}>p_{n}$ for small $n$ and $p_{n}>p_{n}^{*}$ for large $n$. Moreover, these results can be compared with those in the absence of the minimal length scale [58]. Indeed, for small quantum numbers we have $p_{n}>p_{n}(\gamma=0)$ and $p_{n}^{*}>p_{n}^{*}(\gamma=0)$. However, this result becomes reversed for large $n$. It is shown that at a given temperature and $q$, when $\gamma$ increases, the probability functions are also increase for small $n$. But for large $n$ this behavior becomes reverse. As it is known, in zero temperature limit and unlike the BG statistics, the probability function is not equal to unity for the ground state and does not vanish for higher energy states. This behavior is depicted in Fig. 2. Thus, for $T \rightarrow 0$ we find $p_{n}(\gamma=0)>p_{n}$ and $p_{n}^{*}(\gamma=0)>p_{n}^{*}$ for all $n$. Based on Eq. (18), the partition function depends on the temperature, the entropic index $q$, and $\gamma$. As it is shown in Fig. 3. we obtain $Z_{v i b, q^{\prime}}>Z_{v i b, q}$ for $q^{\prime}>q$ and all $T$. Also, we have $Z_{v i b, q}<Z_{v i b, q}(\gamma=0)$ and $Z^{*}{ }_{v i b, q}<Z_{v i b, q}^{*}(\gamma=0)$. For $\gamma_{1}>\gamma_{2}>\gamma_{3}$, it is concluded that for fixed entropic indices and temperatures, we have $Z_{v i b, q}\left(\gamma_{1}\right)<Z_{v i b, q}\left(\gamma_{2}\right)<Z_{v i b, q}\left(\gamma_{3}\right)$. 


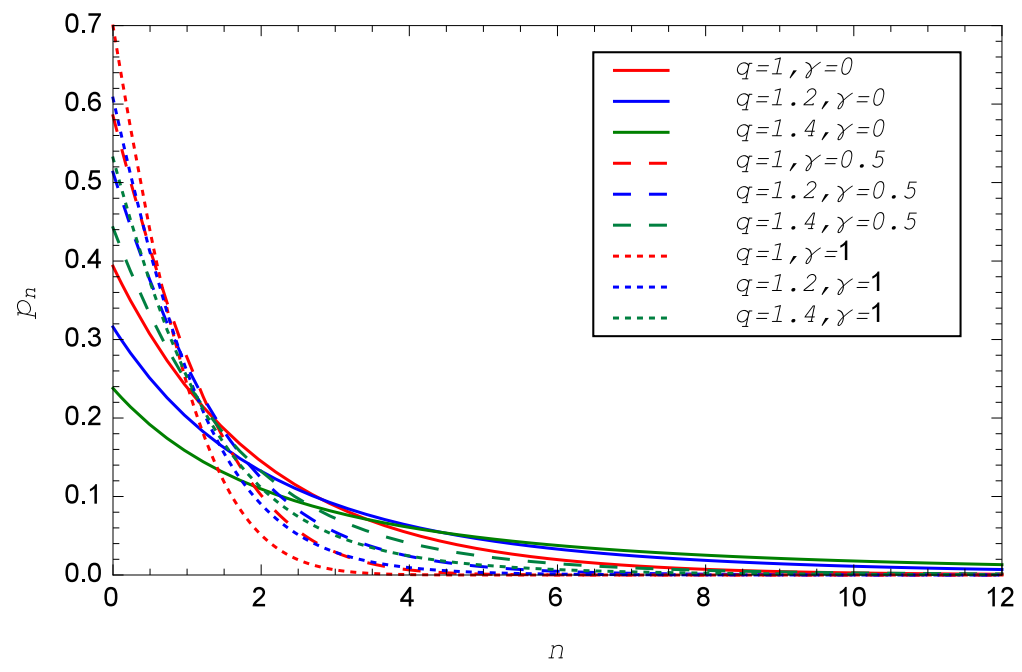

FIG. 1: Comparison between $p_{n}, p_{n}^{*}$ and $p_{n}(\gamma=0), p_{n}^{*}(\gamma=0)$ versus their energy states for $1<q<1.5$ and $k T / \hbar \omega=2$.

Using the properties of the Hurwits zeta function and the definition of the internal energy in the Tsallis statistics

$$
U=-\frac{\partial}{\partial b}\left[\frac{\left(Z_{q}\right)^{1-q}-1}{1-q}\right]=-\frac{1}{\left(Z_{q}\right)^{q}}\left(\frac{\partial Z_{q}}{\partial b}\right)
$$

we find

$$
\begin{aligned}
U= & U_{0}+\frac{\gamma s \hbar \omega[b(q-1) \hbar \omega]^{\frac{q}{1-q}}}{Z_{q}^{q}}\left\{\left(a-\frac{1}{2}\right) \zeta(s, a)-\frac{1}{2} \zeta(s-1, a)-\left(\frac{a^{2}}{2}-\frac{a}{2}+\frac{1}{4}\right) \zeta(s+1, a)\right\} \\
& -\frac{\gamma s[b(q-1) \hbar \omega]^{\frac{1}{1-q}}}{Z_{q}^{q}} \times\left\{\left[\frac{1}{b^{3}(q-1)^{2} \hbar^{2} \omega^{2}}+\frac{\left(a-\frac{1}{2}\right) s}{b^{2}(q-1) \hbar \omega}\right] \zeta(s+1, a)-\frac{(s+1)}{2 b^{2}(q-1) \hbar \omega} \zeta(s, a)\right. \\
& \left.+\frac{\left(-\frac{a^{2}}{2}+\frac{a}{2}-\frac{1}{4}\right)(s+1)}{b^{2}(q-1) \hbar \omega} \zeta(s+2, a)\right\},
\end{aligned}
$$

where $U_{0} \equiv U(\gamma=0)$ is the internal energy corresponds to the absence of the minimal length scale which is given by [58]

$$
U_{0}=\frac{\hbar \omega[(q-1) b \hbar \omega]^{\frac{q}{1-q}} \zeta(s, a)-[(q-1) b \hbar \omega]^{\frac{1}{1-q}} \frac{1}{(q-1)^{2} b^{2} \hbar \omega} \zeta(s+1, a)}{Z_{0}^{q}} .
$$

In contrast to BG statistics, because of the kind of the distribution and the definition for internal energy, in the nonextensive model the internal energy at zero temperature will not be equal to the ground state energy [58]. Also, when the temperature goes to zero, the heat capacity for extensive and nonextensive systems approaches zero but with different slopes. In nonextensive systems we observe an increased energy fluctuation which is due to the increasing the number of accessible states in comparison with extensive ones. The difference in the number of accessible states will be dominated as temperature goes to zero [58].

As it is shown in Fig. 4. for low temperatures we have $U>U^{*}$, but for high temperatures the situation is revered, namely $U^{*}>U$. Note that, this result is not unexpected. In fact, as it is mentioned before, at zero temperature and in Boltzmann-Gibbs statistics for both $\gamma=0$ and $\gamma \neq 0$, the probability of ground state is equal to 1 and it is zero for other excited states. For the nonextensive case, the fluctuations of energy increases and this is because of the 


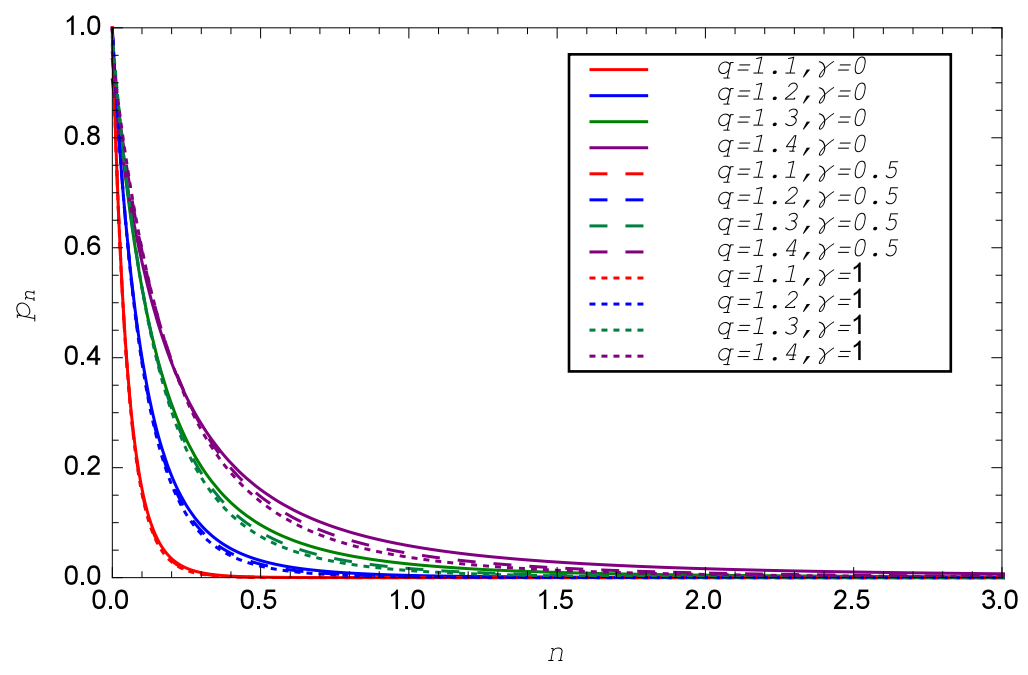

FIG. 2: Comparison between $p_{n}$ and $p_{n}(\gamma=0)$ versus their energy states for $1<q<1.5$ and $k T / \hbar \omega \rightarrow 0$.

increasing of the occupation number of states. In the presence of minimal length, in addition to the fluctuations of the nonextensive statistics, we have fluctuations due to the minimal length scale. Indeed, in nonextensive statistics for both $\gamma=0$ and $\gamma \neq 0$, the probability of ground state is less than 1 for $T \rightarrow 0$. So, in the limit of low temperatures, the nonextensive internal energy must be larger than its corresponding quantity in the absence of this length scale and for the high temperatures the situation becomes reversed. Also, the behavior of internal energy is manifest in this figure for three different values of $\gamma$. For low temperatures, if $\gamma_{1}>\gamma_{2}>\gamma_{3}$ it is concluded that for fixed entropic indices and temperatures, $U\left(\gamma_{1}\right)>U\left(\gamma_{2}\right)>U\left(\gamma_{1}\right)$, but for high temperatures, we obtain different results upon choosing $q$. The reason for the reduction of internal energy in comparing to $\gamma=0$ is a consequence of the reduction of phase space volume due to possible definition of a rescaled $\hbar$. In fact, in the presence of the minimal length uncertainty, the volume of the fundamental cell increases and the number of degrees of freedom reduces consequently [18]. Notice that, in the limit of $q \rightarrow 1$, we obtain the extensive internal energy for quantum harmonic oscillator in the presence of minimal length scale [19].

Another useful thermodynamical quantity is the specific heat capacity. As it is shown in Fig. 5 , in the limit of low temperatures we observe $C_{V}>C_{V}^{*}$. This is due to the fact that at low temperatures for the nonextensive case, the number of accessible states are modified which results in the increase of the heat capacity. However, in the limit of high temperatures we have $C_{V}<C_{V}^{*}$. By comparing the heat capacity in the presence of minimal length $C_{V}$, and its corresponding value in the absence of this minimal length $C_{V}(\gamma=0)$, we find $C_{V}^{*}<C_{V}^{*}(\gamma=0)$ and $C_{V}<C_{V}(\gamma=0)$. Also, for $\gamma_{1}>\gamma_{2}>\gamma_{3}$ we have $C_{V}\left(\gamma_{1}\right)<C_{V}\left(\gamma_{2}\right)<C_{V}\left(\gamma_{3}\right)$. In the nonextensive case unlike the BG case, the heat capacity represents a peak which is also observed for two-level systems [49]. As it is shown in Fig. [5, $C_{V}^{*}-C_{V}$ increases as $q$ grows up. This behavior is due to the relationship between the heat capacity and the internal energy fluctuations [43]. In the limits of $q \rightarrow 1$ and $\gamma \rightarrow 0$ the BG heat capacity for the ordinary quantum harmonic oscillator is recovered.

\section{CONCLUSIONS}

In this paper, we have studied the thermodynamics of quantum harmonic oscillator in the presence of a minimal length uncertainty and in the Tsallis framework. The notion of a nonzero minimal length scale is motivated by various phenomena in high energy physics such as string theory, loop quantum gravity, and black-hole physics. In fact, in the limit of high energies near the Planck energy or equivalently in the limit of small distances near the Planck length, the effects of gravity become dominant which modifies the physical laws. On the other hand the importance of the Tsallis 


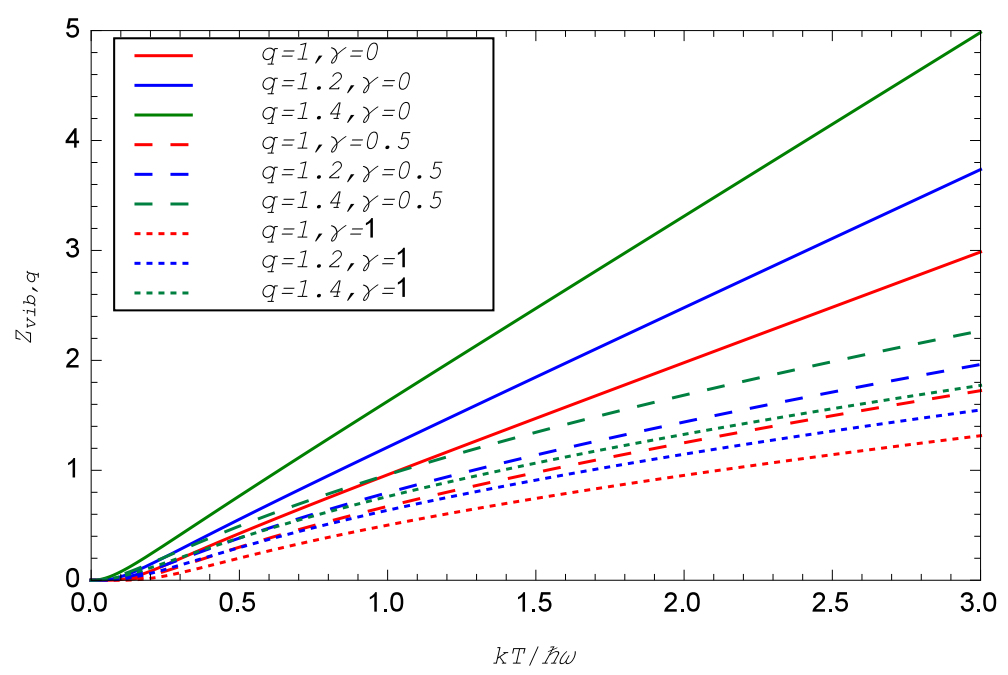

FIG. 3: Comparison between partition functions of extensive and nonextensive quantum harmonic oscillators in the presence of a minimal length scale, $Z_{v i b, q}$, and their corresponding quantum harmonic oscillators without the effects of minimal length scale, $Z_{v i b, q}(\gamma=0)$, versus their temperatures.

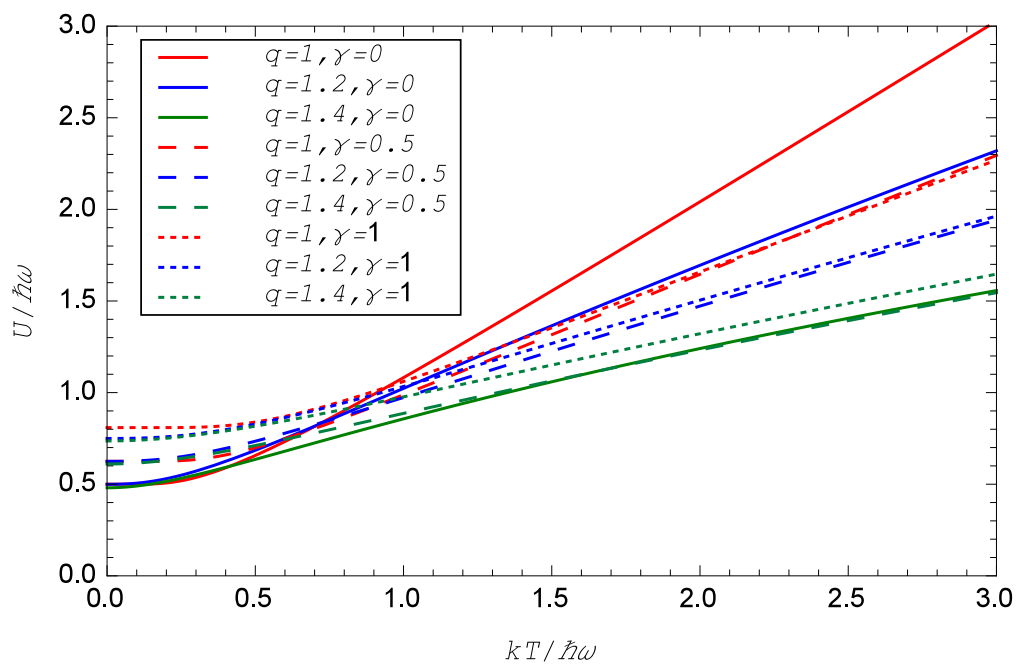

FIG. 4: Comparison between internal energy of extensive and nonextensive quantum harmonic oscillator in the presence of a minimal length scale, $U^{*}, U$ and its corresponding quantum harmonic oscillator without the effects of minimal length scale, $U^{*}(\gamma=0), U(\gamma=0)$, versus their temperatures. 


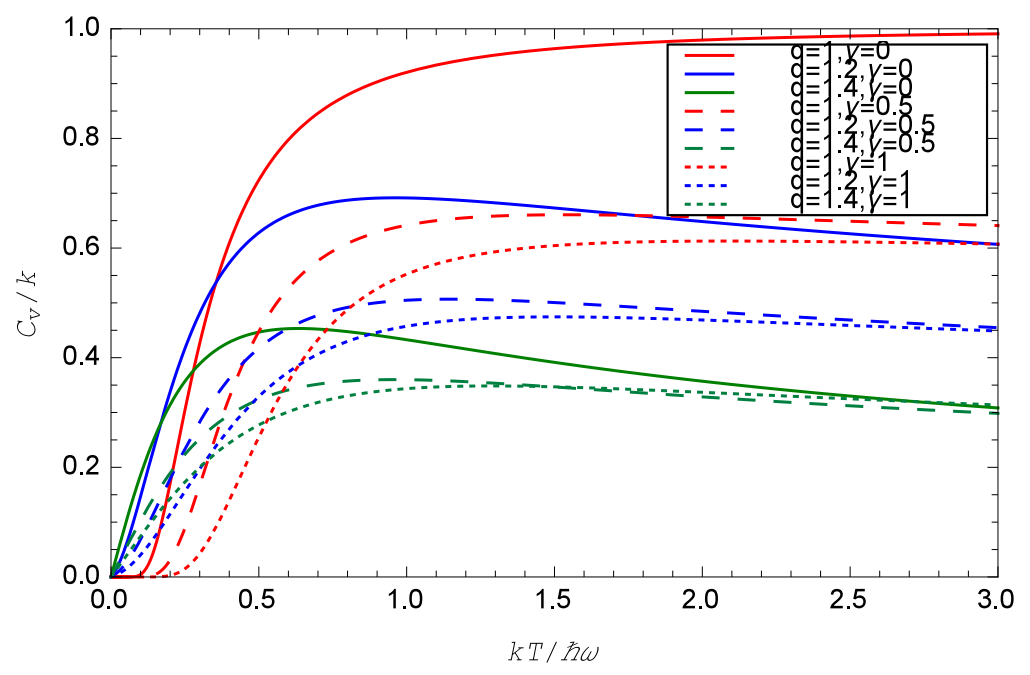

FIG. 5: Comparison between heat capacity of extensive and nonextensive quantum harmonic oscillator in the presence of a minimal length scale, $C_{V}^{*}, C_{V}$ and its corresponding quantum harmonic oscillator without the effects of minimal length scale, $C_{V}^{*}(\gamma=0), C_{V}(\gamma=0)$, versus their temperatures.

statistics as a nonextensive statistical model is due to the fact that the extensive BG statistics has a strong dependence on the initial conditions which is not hold for the most materials. So by considering this two effects, we study the vibrational partition function, probability function, internal energy, and the heat capacity analytically for $1<q<\frac{3}{2}$. It is shown that the nonextensive internal energy is larger than its extensive counterpart for low temperatures and vise versa for the high temperatures. This behavior is related to the different definitions of the internal energy in the extensive and nonextensive statistics and the dependence of the probability function to the entropic index. It is also shown that $\frac{\partial Z_{v i b, q}}{\partial T}>0$ and $Z_{v i b, q^{\prime}}>Z_{v i b, q}$ for $q^{\prime}>q$. Then, the heat capacity is studied and it is indicated that at high temperatures the nonextensive heat capacity is smaller than the extensive one and this is because of the statistical correlations in the nonextensive statistics. At low temperatures, due to the number of accessible states a larger fluctuation is expected. We also studied the behavior of the probability function in the presence of a minimal length scale for two different temperatures and found that for the nonextensive statistics, the probability of finding the system at the ground state is not equal to unity even at the zero temperature. Finally, in the limit of $q \rightarrow 1$, the corresponding Boltzmann-Gibbs results in the presence of the minimal length uncertainty is recovered. These results could also shed lights on the black-body radiation with minimal length and in Tsallis thermodynamics.

\section{References}

[1] G. Veneziano, Europhys. Lett. 2, 199 (1986).

[2] D. Amati, M. Ciafaloni and G. Veneziano, Phys. Lett. B 216, 41 (1989).

[3] D. Amati, M. Ciafaloni and G. Veneziano, Phys. Lett. B 197,81 (1987).

[4] D. Amati, M. Ciafaloni and G. Veneziano, Int. J. Mod. Phys. A 7, 1615 (1988).

[5] D. Amati, M. Ciafaloni and G. Veneziano, Nucl. Phys. B bf 347, 530 (1990).

[6] D.J. Gross and P.F. Mende, Nucl. Phys. B 303, 407 (1988).

[7] K. Konishi, G. Paffuti and P. Provero, Phys. Lett. B 234, 276 (1990). 
[8] S. Capozziello, G. Lambiase and G. Scarpetta, Int. J. Theor. Phys. bf 39, 15 (2000).

[9] L.J. Garay, Int. J. Mod. Phys. A 10, 145 (1995).

[10] A. Kempf, G. Mangano and R.B. Mann, Phys. Rev. D 52, 1108 (1995).

[11] S. Doplicher, K. Fredenhagen, and J.E. Roberts, Phys. Lett. B 331, 39 (1994); Comm. Math. Phys. 172 , 187 (1995).

[12] H.S. Snyder, Phys. Rev. 71, 38 (1947).

[13] A. Connes, Noncommutative Geometry (Academic Press, New York, 1994); J. Math. Phys. (N.Y.) 41, 3832 (2000).

[14] J.C. Varilly, An Introduction to Noncommutative Geometry (EMS Series of Lectures in Mathematics) (European Mathematical Society, Zurich, 2006).

[15] M.R. Douglas and N.A. Nekrasov, Rev. Mod. Phys. 73, 977 (2001).

[16] T.V. Fityo, Phys. Lett. A 372, 5872 (2008).

[17] K. Nozari, A.S. Sefidgar, Chaos, Solitons and Fractals 38, 339 (2008).

[18] K. Nozari, S. H. Mehdipour, Chaos, Solitons and Fractals 32, 1637 (2007).

[19] P. Pedram, Phys. Rev. D 85, 024016 (2012).

[20] G.A. Camelia, M. Arzano, Y. Ling and G. Mandanici, Class Quant Grav, 23, 2585 (2006).

[21] S.K. Rama, Phys. Lett. B 519, 903 (2001).

[22] B. Vakili, M.A. Gorji, J. Stat. Mech. P10013 (2012).

[23] M. Abbasiyan-Motlaq and P. Pedram, J. Stat. Mech. P08002 (2014).

[24] C. Beck, Physica A 277, 115 (2000).

[25] H.Y. Zhang, X. Gu, X.H. Zhang, X. Ye and X.G. Gong, J. Phys. Lett. A 331, 332 (2004).

[26] H.M. Gupta, J.R. Campanha and S.J. Schinaider, Physica A 387, 6745 (2008).

[27] A. Razdan, Pramana J. Phys. 68, 61 (2007).

[28] S. Tonga, Physica A 305, 619 (2002).

[29] C. Tsallis, J. Stat. Phys. 52, 479 (1988).

[30] W.C. Saslaw, Gravitational physics of stellar and galactic systems (Cambridge University Press, Cambridge, 1985); J. Binney and S. Tremaine, Galactic dynamics (Princeton University Press,Princeton, 1987).

[31] H. Risken, The Fokker-Planck equation (Springer-Verlag, Berlin, 1984), page 9.

[32] M.O. Caceres, Braz. J. Phys. 29, 125 (1999).

[33] C. Tsallis, Brazilian Journal of Physics, 29, 1 (1999).

[34] Y.H. Taguchi and H. Takayasu, Europhys. Lett. 30, 499 (1995).

[35] E. Montroll and M.F. Shlesinger, J. Stat. Phys. 32, 209 (1983); M.F. Shlesinger, G.M. Zaslavsky and U. Frisch, Levy ights and related topics in Physics (1995).

[36] X.P. Huang and C.F. Driscoll, Phys. Rev. Lett. 72, 2187 (1994).

[37] I. Koponen, Phys. Rev. E 55, 7759 (1997).

[38] D.C. Clayton, Nature 249, 131 (1974).

[39] N.A. Bahcall and S.P. Oh, Astrophys. J. 462, 49 (1996).

[40] J.M. Liu, J.S. De Groot, J.P. Matte, T.W. Johnston and R.P. Drake, Phys. Rev. Lett. 72, 2717 (1994).

[41] J. Maddox, Nature 365, 103 (1993).

[42] Z.H. Feng and L.Y. Liu, Physica A 389, 237 (2010).

[43] L. Liyan and D. Jiulin, Physica A 387, 5417 (2008).

[44] H.H. Aragao-Rego, D.J. Soares, L.S. Lucena, L.R. da silva, E.K. Lenzi and K.S. Fa, Physica A 317,199 (2003).

[45] J. Carrete, L.M. Varela and L.J. Gallego, Physica A 387, 6752 (2008).

[46] I. Fukuda and H. Nakamura, J. Phys. Chem. B 108, 4162 (2004).

[47] H. Hasagawa, Phys. Rev. E 80, 011126 (2009).

[48] R. Chakrabarti, R. Chandrashekar and S.S. Nina Mohammed, Physica A 387, 4589 (2008).

[49] C. Tsallis, R.S. Mendes and A.R. Plastino, Physica A 261, 543 (1998).

[50] E.K. Lenzi and R.S. Mendens, Phys. Lett. A 250, 270 (1998).

[51] E.K. Lenzi, R.S. Mendes, L.R. da Silva and L.C. Malacarne, Phys. Lett. A 289, 44 (2001).

[52] P. Sadeghi, S. Khademi and A.H. Darooneh, Phys. Rev. A 86, 012119 (2012).

[53] S. Abe and A.K. Rajagopal, Phys. Rev. A 60, 5 (1999).

[54] E.K. Lenzi, L.C. Malacarne and R.S. Mendes, Physica A 278, 201 (2000).

[55] A. Ghosh and P. Chaudhuri, Int. J. Theor. Phys. 39, 10 (2000).

[56] A.M. Nassimi and G. Parsafar, J. Iran. Chem. Soc. 6, 341 (2009).

[57] S. Abe, Physica D 193, 84 (2004).

[58] E. Keshavarzi, M. Sabzehzari and M. Eliasi, Physica A 389, 2733 (2010).

[59] C. A. Mead, Phys. Rev. B 135, 849 (1964).

[60] M. Maggiore, Phys. Lett. B 304, 65 (1993).

[61] E. Witten, Phys. Today 49, 24 (1996).

[62] F. Scardigli, Phys. Lett. B 452, 39 (1999).

[63] R.J. Szabo, Phys. Rep. 378, 207 (2003).

[64] P. Pedram, Physica A 391, 2100 (2012).

[65] J. Magueijo and L. Smolin, Phys. Rev. Lett. 88, 190403 (2002).

[66] J. Magueijo and L. Smolin, Phys. Rev. D 71, 026010 (2005).

[67] J.L. Cortes and J. Gamboa, Phys. Rev. D 71, 065015 (2005).

[68] A.F. Ali, S. Das and E.C. Vagenas, Phys. Lett. B 678, 497 (2009). 
[69] S. Das, E.C. Vagenas and A.F. Ali, Phys. Lett. B 690, 407 (2010).

[70] P. Pedram, Phys. Lett. B 710, 478 (2012).

[71] I.S. Gradshteyn and I.M. Ryzhik, Table of Integrals, Series and Products, 5th ed., Academic Press, New York, (1994).

[72] E.M.F. Curado and C. Tsallis, J. Phys. A 24, 69 (1991). 